\title{
The Study of Training the Innovative Agriculture and Forestry Talents on the Basis of the Teaching Management System
}

\author{
Zhangwen Hui ${ }^{1, a}$, Niehai Yan ${ }^{2, b}$, Lide Cai ${ }^{1,}$, Zhaixue Feng ${ }^{1, \text { a }}$ \\ ${ }^{1}$ Traffic college, Northeast Forestry University, Harbin 150040, China \\ ${ }^{2}$ Harbin Far East Institute of Technology, Harbin 150025, China \\ aemail: lidecai2008@sina.com, b47738036@qq.com
}

Keywords: forestry; innovative talents; teaching managemen

\begin{abstract}
Cultivating innovative talents is a rather concerned and major problem not only in some Chinese basic work, that is, to become an innovative country and a human resources powerful nation and to bring about a great rejuvenation of the Chinese nation, but also in National Medium and Long-term Education Reform and Development Plan Outline. As a key university in China, Northeast Forestry University, which located in the forest industry zone of Heilongjiang province with distinct characteristics in forestry, has carried out education teaching reform with more efforts in recent years, starting from various reform works and positively exploring the training methods of innovative talents. So it has achieved initial results and has drawn wide attention from educational world, especially from agriculture and forestry educational institutions. For the past few years, Northeast Forestry University did a lot of work in building rules and regulations of teaching management. From the perspective of building teaching management system to cultivate innovative talents majored in forestry, this paper mainly sets forth its role with the purpose to establish a sound system safeguard for the university to train new forestry talents in following years and meanwhile offer some reform references in cultivating innovative talents for other universities and colleges .
\end{abstract}

\section{Introduction}

In The Outline of National Medium-and-long-term Educational Reform and Development Planning, the reform of personnel training system is at the top of list of the system reform. Northeast Forestry University has kept exploring in terms of cultivating innovative talents in recent years, especially it has achieved outstanding results in cultivating innovative forestry talents in the premise of bringing forestry superiority into full play. Now NEFU is a university that is committed to the construction of innovational platform of national 985 preponderant disciplines and also an experimental university carried out the plan of education and training for national outstanding engineers, which is an experimental project of national education system reform --- to plan cultivating the very best innovative talents of forest resources. In terms of teaching management system construction, NEFU reformed rationally, stuck to prominence of major points, advance on the whole, traditional inheritance and bold innovation, and built up the effective and useful teaching management system with coordinative contents, strict procedure and complete supporting, which has played an important role in cultivating innovative talents.

\section{The Teaching Management System-a Guarantee of Setting up a Good Professional Platform to Train the Innovative Forestry Talents}

Specialty construction is a foundation to breed and promote the core competitiveness of colleges and universities, only can specialty construction bring about the high quality and high levels of innovative talents[1]. The colleges and universities should positively explore to set up the discipline professional mechanism according to the social need, which can help to realize the good coordination between the discipline construction and economic and social development. In the teaching management system of the university, the key point of all the systems focused on how to promote the level of specialty construction and the quality of training talents. In such a series of 
systems set up by NEFU as "The Implementing Measures of Gradually Strengthening the Specialty Construction in NEFU" and "The Development Scheme of the Key Undergraduate Course during the Period of the Twelfth Five-year Plan in NEFU", the fact that the specialty construction should be developed step by step according to the plan is emphasized definitely in order to build up a number of high-level and high quality courses with distinct characteristics in the future. Under the guide of the teaching management system, our university stuck to the connotative development and then realized the further development through constructing specialty, strengthening the teaching management, deepening the teaching reform, guiding the courses, the teachers, the teaching models and the teams of professional teaching and building up and reforming the conditions of practice teaching according to the demands such as the accurate level positioning, the advanced training model, the distinctive specialty and good talent. As a result, it set up such undergraduate course constructing pattern with the forestry as the superiority and forestry engineering as the characteristics and coordinative development of multi-disciplines. There are nine national characteristic specialties, twenty-six key provincial specialties and thirty key school-class specialties in NEFU, among which the forestry specialties are all national characteristic specialties or key provincial specialties, which established the good professional platform to train the innovative talents of the forestry field, promoted the whole level of the innovative forestry talents and initiatively adapted to the demands of the national education strategies and the economic development of industries.

\section{The Teaching Management System- a Guarantee of Constructing Multi-layer Training Mode to Train the Innovative Forestry Talents}

In the process of the long-term teaching management practice, NEFU persists in and enriches the school-running characteristics and continuously explores the training model of the innovative forestry talents in order to build up the training system of the multi-discipline, multilevel and multi-type innovative forestry talent to meet the need of the social and economic development. Our university depends on these three specialties, that is, the forestry, the forest resources protection and recreation and wild animals and nature reserve management, to carry out the training model reform of the outstanding and innovative forestry talents, which is an experimental program of the national education system reform; depends on wood science and engineering and civil engineering to put the training model reform of the outstanding talents into effect; depends on the artistic designing (interior and furniture designing) to carry out the training model reform of the innovative and entrepreneurial talents; depends on the national Science basic scientific research and teaching personnel training base and the national base of fife science and technology education to conduct the training model reform of the basic subject talents, initially forming the four-level training system of the innovative forestry talents. At the same time, our university gives full play to two national innovative experimental plot of personnel training model such as wildlife and natural reserves management and artistic designing (interior and furniture designing) and six school-level in forestry such as chemical engineering of forest products and forest engineering and forest exploitation concerning foreign affairs to expand the training way of the innovative forestry talents. In the personnel training model reform, the teaching management system should continuously follow up, taking a national experimental reform of the education system in NEFU--- the training model reform of the outstanding and innovative forestry talents for example, our university has set up a series of management system such as "The Personnel Training Program of Opportunity Class in Forest Reserves" and "The Management Methods of Opportunity Class in Forest Reserves" and then conduct the scientific planning and the beneficial attempt from the educational idea, the operating mechanism, the innovative exploration and the reform of practice teaching, accumulating valuable experience for fully and deeply promoting the training of the innovative forestry talents.

\section{Curriculum System to Train the Innovative Forestry Talents}

Curriculum construction is a foundation and a central part of discipline construction and 
specialty construction, a core of the reform of the teaching contents, and an important method and a basic way to improve the teaching quality[2]. In view of the teaching management of training the innovative talents, our university set up such systems as The Planning of Curriculum Construction in NEFU, The Management Method of the Key Course Construction in NEFU and A Number of Suggestions for Fully Developing the Reform of the Teaching Methods and Exams in NEFU in order to provide system guarantee for setting up the scientific curriculum system. In the process of the curriculum construction, our university boasts seven national essential courses, three national open courses of essential video and one national bilingual demonstration course, which are all core curriculums of the forestry. Among 29 provincial essential courses, 24 are the core courses of the forestry and 32 in 101 school-level essential courses. At present, our university continuously strengthens the curriculum construction consisted of open courses of essential video, essential courses of resource sharing, high-quality courses, network courses and content-based courses, because the curriculum construction can promote constantly the reform of teaching methods and teaching means and optimize and innovate the teaching contents to further perfect the curriculum system of training the innovative forestry talents.

\section{The Teaching Management System-a Guarantee of Providing Excellent Teachers to Train the Innovative Forestry Talents}

In the development of the university, we always believe that teachers are the most important educational resources[3]. Teachers are one of the fundamental factors that affect the teaching quality and the level of training talents in the process of education and teaching[4]. For example, in the practical work of improving the teaching level, our university sets up The Enforcement Regulation of Training and Higher Learning for the Undergraduate Teachers in NEFU and The Temporary Provisions for the Supervisor System and Assist-class System of Young Teachers in NEFU, which helped the university produce the five-in-one training mode consisted of the pre-job training of full-time teachers, the school-based teacher training, the professional training, the backbone training and the special training. The teachers training has taken shape a new pattern of multilevel, multiform and multipath in colleges and universities. In the meantime, our university depends on the plan of training the various levels of talents set by the Ministry of Education to carry out the personnel training plan of star teachers and gifted persons through vigorously supporting the teachers' training, higher learning and visit study at home and abroad, and introducing the outstanding talents from our own countries and overseas. Our university strengthens the service and management of the teachers, establishes the sound teachers' evaluation mechanism and enhances the contraction of the academic teams so that it can be sure to provide the excellent teachers for training the innovative forestry talents. For instance, our university boasts two academicians of the Chinese Academy of Engineering, six double-hired academicians who are the well-known professors in the forestry field, six of seven professors who are awarded the provincial star teachers are all forestry professors. Meanwhile, there are two national teaching teams and five provincial ones that are all from the forestry field in the university, which actively lead the young and middle-age teachers to throw themselves into the teaching of the undergraduate, improving definitely its teaching quality. The construction of the star teachers and teaching teams strongly promoted the improvement of the whole quality of the teachers and has made great contribution to boost the training of the innovative forestry talent.

\section{The Teaching Management System -a Guarantee of Offering Abundant Practice to Train the Innovative Forestry Talents}

The social demand is a motive power for the development of the colleges and universities, so the forestry modernization is a powerful driving force and a rare opportunity for the development of the forestry schools and colleges[5]. In order to fully improve the level of running a school and strengthen the students' social practice ability, our university encourages the school-enterprise cooperation in the overall way, makes efforts to realize the interaction with the local development, 
and deepens constantly the reform of the teaching system of experiment and practice to fully improve its teaching level. For the sake of strengthening the practice teaching, our university set up a series of systems such as The Management Method of the Innovative Credit of the Undergraduate Students in NEFU, The Construction and Management Method of the Practice Base of Teaching and Scientific Research Outside School in NEFU and The Management Method of the Plan of the Innovative Entrepreneurial Training in NEFU, providing the relatively impeccable system guarantee for the practice of the innovative forestry talents and ensuring the quantity and quality of building up the teaching practice base of the undergraduate students. It's worth mentioning that our university boasts three national demonstration center of experimental teaching that are all the forestry and eight provincial ones, among them, five are the forestry. It also boasts the personnel training base of agricultural science and education and the educational center of the engineering practice of the forestry engineering group in Heilongjiang and one in Jilin. It boasts ten practice bases on campus and 210 ones outside school such as the experimental tree farm in Harbin, Mao'er Mountain experimental tree farm (Mao'er Mountain National Forest Park), Liangshui experimental tree farm (Liangshui National Natural Reserves) and so on. There are a good deal of rare and precious species in the experimental tree farm, which is the paradise of teaching, scientific research and practice. It is this kind of advantaged resource superiority that any other forestry schools or colleges do not have in China. In recent years, the students' thesis of the forestry specialty in our university such as the forestry, the forest conservation and recreation and so on are all first-hand projects of the scientific research so that the students' practice ability improved greatly, forming the characteristics of the practice and educating in NEFU.

\section{Acknowledgment}

In this paper, A Special Funds for the Basic Scientific Research of the Central Colleges and Universities (Project No. DL10CC19); A Project to Reform the Higher Education and Teaching of Heilongjiang in 2011; The Scientific Research Subject of Higher Education of the Twelfth Five-year Plan Conducted by the Branch of Higher Education of China's Forestry Education Society in 2011; The Annual Subject of the Twelfth Five-year Plan Conducted by Heilongjiang Education Science in 2011 (Project No. GBD1211006); The Education Research Subject of Northeast Forestry University

(Project No. DGY2010-01); The Monographic Research Program of Northeast Forestry University in 2012.

\section{References}

[1] Bie Guorong. To Reform the Teaching Management System of Colleges and Universities with the Modern Idea [J]. The Higher Education in China,2007,(20):45 46.

[2] Gao Guozhong, Yu Wen. The Rethinking of Power Construction with Higher Education from the Perspective of the Historical Position of Higher Education [J]. Heilongjiang Researches on Higher Education, 2012,(4):19 21.

[3] Li Qingfeng. To Strengthen the Curriculum Reform of Colleges and Universities in Order to Improve the Personnel Training [J]. The Higher Education in China,2012,(7):29 30.

[4] Yang Chuanping. To Enhance the Core Competitiveness of Colleges and Universities through Taking Full Advantages and Cultivating the Characteristics [J]. The Higher Education in China, 2010,(21):32 33 .

[5] Huang Naiwen. The Research of the Teaching Management System Reform of Colleges and Universities on the Basis of Quality -oriented Education and Innovatin Capability [J]. The Adult Education in China,2010,(4):23 24 